\title{
Beneficios de los principios de Tennis Play and Stay en el desarrollo físico de los tenistas jóvenes
}

\author{
Fernando Vilches
}

\section{RESUMEN}

El presente artículo tiene como objetivo analizar las ventajas que brindan los principios del Play and Stay en el desarrollo físico de los jóvenes tenistas. Los años iniciales del niño incorporado al deporte son muy importantes ya que permiten desarrollar la base motora que luego le otorgara importantes beneficios en su posterior desarrollo.
Palabras clave: Play and Stay, desarrollo motor, aprendizaje significativo, método integral

Recibido: 19 Dic 2016

Aceptado: 20 Mar 2017

Autor correspondiente:

Fernando Vilches

Correo electrónico:

fervilches@hotmail.com

\section{INTRODUCCIÓN}

El tenis es un deporte complejo que requiere coordinación, percepción, toma de decisiones y habilidades específicas. Si pensamos en el grupo de niños menores de 10 años debemos considerar que se dan en ellos una serie de procesos físicos que afectan directamente a lo que el niño es capaz de hacer. Es fundamental que el entrenador entienda que los procesos de crecimiento y maduración del niño pueden limitar la capacidad que tiene para realizar una tarea. Durante muchos años la presentación de las actividades a los niños era similar a la de un adulto sin tener en cuenta su crecimiento y maduración.

Por suerte la introducción del Play and Stay permite que los alumnos puedan experimentar un proceso de aprendizaje adaptado a las necesidades psicomotoras del niño, desarrollando un aprendizaje más significativo.

Entre los beneficios más importantes podemos destacar los siguientes puntos clave:

\section{Crea un entorno positivo para el aprendizaje} favoreciendo el ajuste perceptivo motriz:

Desde el punto de vista motor el niño se encuentra en un proceso de continuo crecimiento atravesando un periódico ajuste perceptivo motriz en función del espacio, del tiempo y en relación a los objetos (Pelota y Raqueta). La adaptación de estos ejes en la progresión de los ejercicios ayudará al niño a mantener un reto óptimo en las actividades que realiza, permitiendo atravesar cada etapa con el grado de dificultad adecuada.

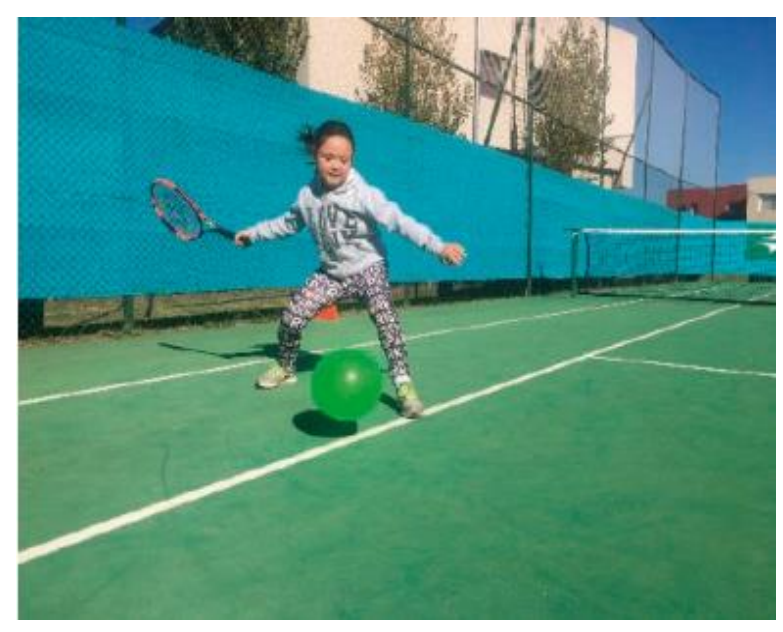

Los principales ejes motrices que permiten un ajuste adecuado son:

\footnotetext{
- El propio cuerpo

- El espacio

- El tiempo

- Objeto pelota
} 


\section{- Objeto raqueta}

A raíz de lo mencionado anteriormente es fundamental que el profesor identifique el grado de complejidad adecuado en cada eje para favorecer un desarrollo armónico y progresivo. Diseñar canchas creativas con un gran número de situaciones problema a resolver será crucial para el dominio futuro de situaciones complejas.

\section{Permite el desarrollo de las habilidades motoras básicas:}

Todos los que trabajamos alguna vez con jóvenes tenistas hemos hecho hincapié en el tema de la habilidad de nuestros jugadores. Cuantos mayores sean las experiencias motrices de los niños mayores serán las posibilidades de este mismo para el aprendizaje de habilidades específicas.

Incluir actividades que desarrollen habilidades motoras como el correr, lanzar, enviar o recibir le permitirán al niño ampliar las herramientas motrices con las que en un futuro podrá resolver situaciones problema con éxito.

Una estructuración armónica y organizada del esquema corporal y de las habilidades motoras básicas en diversas situaciones y con grados de dificultades crecientes influye radicalmente en el proceso de movimiento y ejecución de los golpes.

Es muy importante que el profesor identifique que el niño pasará por distintos estados de aprendizaje de dichas habilidades:

- Estadio temprano: El niño parece no dominar el ejercicio, suelen observarse un gran número de errores (el error forma parte del aprendizaje)

- Estadio refinado: El niño presenta un nivel básico de dominio de la habilidad y puede lograr el éxito en algunas oportunidades (dar tiempo de práctica es muy importante en este estadio)

- Estadio fluido: El niño realiza automáticamente la habilidad ampliando su repertorio de ejecución (la clave de este estadio es generar cierta inestabilidad aumentando el grado de dificultad, generando nuevos aprendizajes)

\section{Favorece la incorporación de movimientos específicos} poco utilizados en la vida cotidiana

Hay una tendencia a preocuparse porque el niño maneje rápidamente la raqueta centrando la atención en la misma y no en los movimientos que produce el cuerpo para moverla. Es muy importante que el niño tenga un buen manejo de su cuerpo y de los movimientos ya que "es el cuerpo el que mueve la raqueta" y no en sentido inverso. Hay un gran repertorio de movimientos específicos que requieren un gran control corporal en el tenis y que el niño a edades tempranas no suele utilizarlos en acciones de la vida cotidiana.
Un ejemplo de dichos movimientos específicos podría ser: - Realizar dos movimientos opuestos para una misma acción con el tren inferior y con el tren superior (Ej: Tren inferior ejecuta los desplazamientos mientras que el tren superior gira y rota hacia atrás para producir el golpe en un Drive)

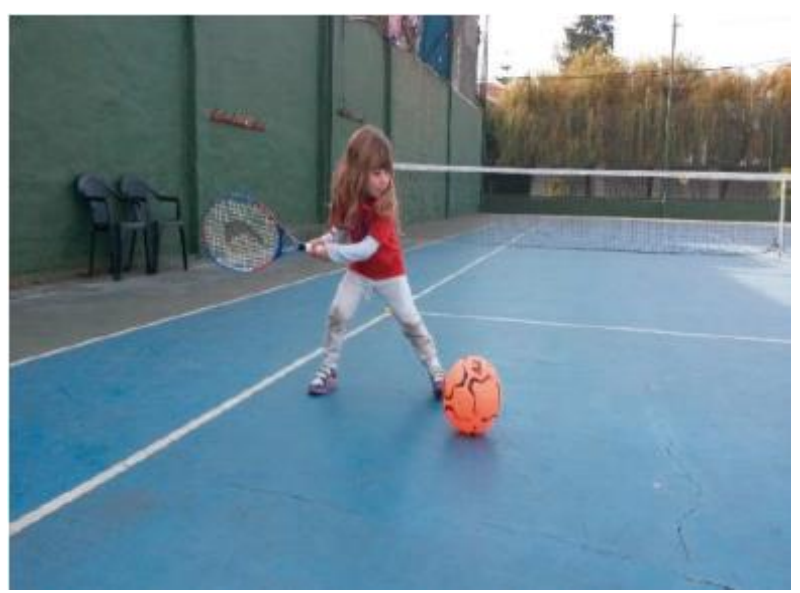

- Realizar acciones en un eje distal al centro de gravedad por encima de la cabeza (Ej: Saque - smash)

- Utilizar de manera coordinada ambos brazos para una misma acción (Ej: Revés a dos manos)

- Utilizar el brazo no dominante para realizar una acción que requiere precisión (Ej: lanzamiento en el saque)

La estimulación previa de estos movimientos a través de juegos, actividades o ejercicios beneficiará en gran medida al futuro aprendizaje de la técnica. Un espacio adaptado de Play Tenis a través de la adaptación de las canchas y las pelotas será el medio ideal para potenciar estas prácticas motoras.

\section{Es el ámbito ideal para potenciar el desarrollo de las capacidades coordinativas:}

La coordinación motriz cumple un rol fundamental en estas etapas del aprendizaje ya que podríamos considerarla como una capacidad integradora de todas las áreas y que en este estadio se constituye dentro de un periodo muy sensible para su desarrollo. Es decir la coordinación actúa como nexo entre todos los elementos que intervienen en un movimiento, integra lo perceptivo, el proceso de toma de decisiones y lo motor.

Potenciar cada una de estas capacidades permitirá al niño resolver situaciones motrices con cierto grado de complejidad y con aciertos en la toma de decisiones.

El desarrollo de la coordinación le permite al niño entre otras cosas:

- Desarrollar la memoria motriz.

- Favorecer a la creatividad. 
- Asimilar nuevos patrones de movimiento.

- Fundamentar el aprendizaje de aspectos técnicos.

- Predisposición cognitiva para el aprendizaje.

- Economizar el esfuerzo.

- Mejorar la atención.

Una vez que el niño va formando este "archivo" de movimientos, es solo cuestión de empezar a combinarlos. Invertir tiempo en el desarrollo del sentido del equilibrio, el tono muscular apropiado para una buena postura, la propiocepción y la sensibilidad de su cuerpo antes situaciones nuevas $o$ ante pelotas en movimiento, permitirán el crecimiento de un deportista más completo que sea capaz de adaptarse a situaciones inestables e imprevistas muy frecuentes en el tenis moderno.

El jugador que adquiere el hábito de movimientos coordinados a edades tempranas podrá en un futuro continuar trabajando su coordinación y agregando dificultades a medida que desarrolla aptitudes nuevas. Podrá así realizar estas acciones más rápidas (velocidad), durante tiempos más prolongados (resistencia) y con mayor fuerza.

\section{Hacia un concepto integrador:}

Cada uno de los aspectos mencionados anteriormente favorece al desarrollo de un deportista completo. El escenario en el que se llevan a cabo las clases de Play and Stay son el mejor ámbito para poder implementar la idea de un método integral de las áreas en las que la técnica, la táctica, lo motor y lo cognitivo integren una misma sesión en función de objetivos determinados.

Dentro de este concepto integrador no podemos dejar de incluir el aprendizaje y la práctica de otros deportes.

Entre las ventajas más importantes de la inclusión de otros deportes o actividades que incluyan problemáticas propias podemos considerar:

- Favorecen al desarrollo físico en general, la capacidad de adquirir una habilidad, coordinación, movilidad y desarrollo de los sentidos.

- Resolver situaciones problemáticas propias de otros deportes desarrolla una notable mejoría en el proceso de resolución de problemas y toma de decisiones.

- Genera motivación en los niños y permite compartir con otros la sensación de equipo, pertenecer a un grupo, identificarse y ser solidario dentro de un deporte individual.

\section{CONCLUSIÓN}

El sistema de enseñanza a través del Play \& Stay es sin dudas el mejor escenario para el desarrollo de aquellas habilidades que formarán parte de la base motora del niño y que le permitirán resolver las situaciones que se le presenten con éxito en el deporte.

Saber "que" y "como" responder, en deportes como el tenis que requieren gran velocidad de reacción, ejecución técnica de precisión y gran variabilidad en la toma de decisiones son razones más que suficientes para apoyar nuevos planteamientos metodológicos que pongan en práctica todos los conceptos citados en el presente artículo.

\section{REFERENCIAS}

Anton, J. y col. (1989). Entrenamiento deportivo en la edad escolar. Colección Uniesport. Cádiz.

Apuntes de la (2000) Cátedra Educación Física Infantil Profesorado de Educación Física F.W. Dickens INEF Nro. 2

Bariles Reynoso. (2008) El Tenis y la coordinación motriz. Gráficamente Ediciones.

Bompa. T.O. (2000) Total Training four Young champions. Champaign, IL: Human Kinectics.

Crespo, M. y Miley, D. (1998). Advanced Coaches manual. Federacion Internacional de Tenis.

Crespo, M., y Reid, M. (2009). Entrenamiento de Tenistas Iniciantes e intermedios: Manual del programa de formación de entrenadores de la ITF. ITF. International Tennis Federation.

Forti, L. (1992). La formación del tenista completo. Editorial Paidos.

Giraldes, M. (1998). La gimnasia Formativa en la Niñez y Adolescencia. Editorial Stadium. Giraldes, M. (1998).Buenos Aires Giraldes, M. (2000). Didáctica de una cultura de lo corporal. Editorial del autor.

Le Boulch, J. (1981) La educación del movimiento en la edad escolar. Paidós Ibérica.

Reid. M, Quinn. A, Crespo. M, (2003). Fuerza y Condición Física para Tenis ITF, London.

Segal, F. (1996). Sistema Analítico Formativo. 
CONTENIDO ITF ACADEMY RECOMENDADO (HAZ CLICK ABAJO)

\section{ITF Academy}

Derechos de Autor (c) 2017 Fernando VilcheS.

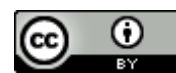

Este texto está protegido por una licencia CreativeCommons 4.0 .

Usted es libre para Compartir -copiar y redistribuir el material en cualquier medio o formato- y Adaptar el documento -remezclar, transformar y crear a partir del material- para cualquier propósito, incluso para fines comerciales, siempre que cumpla la condición de:

Atribución: Usted debe dar crédito a la obra original de manera adecuada, proporcionar un enlace a la licencia, e indicar si se han realizado cambios. Puede hacerlo en cualquier forma razonable, pero no de forma tal que sugiera que tiene el apoyo del licenciante o lo recibe por el uso que hace de la obra.

$\underline{\text { Resumendelicencia - Textocompletodelalicencia }}$ 